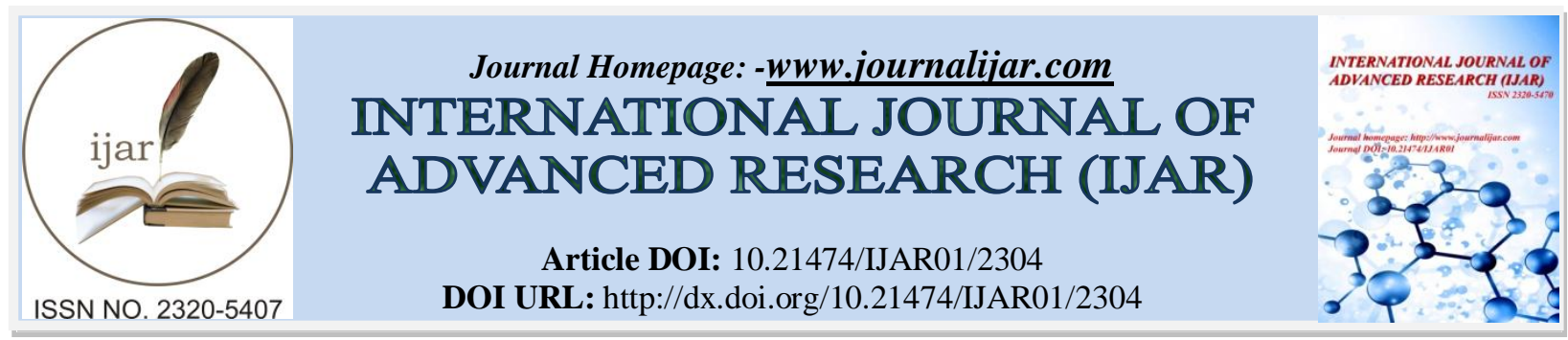

RESEARCH ARTICLE

\title{
DEVELOPING AN ECONOMIC SYSTEM THAT CAN GIVE A BLIND PERSON BASIC SPATIAL AWARENESS AND OBJECT IDENTIFICATION.
}

\author{
Debargha Ganguly.
}

\section{Manuscript Info}

Manuscript History

Received: 29 September 2016

Final Accepted: 30 October 2016

Published: November 2016

\begin{abstract}
Project BATEYE fundamentally uses an ultrasonic sensor mounted on to a wearable pair of glasses that measures the distance to the nearest object and relays it to an Arduino board. The Arduino board then processes the measurements and then plays a tone $(150-15000 \mathrm{~Hz})$ for the respective distance $(2 \mathrm{~cm}$ to $4 \mathrm{~m})$ till the data from the second ultrasonic pulse (distance) comes in, and then the same process gets repeated. This cycle is repeated almost every 5 milliseconds. The person hears sound that changes according to the distance to the nearest object. The head provides a 195-degree swivel angle and the ultrasonic sensor detects anything within a 15-degree angle. Using systematic, cognitive and computational approach of neuroscience, with the hypothesis that the usage of the occipital lobe of blind people goes into processing other sensory feedback., and using the brain as a computational unit, the machine relies on the brain processing the tone produced every $14 \mathrm{mS}$ to its corresponding distance and producing a soundscape corresponding to the tones and the body navigating using the same.During experimentation, the test subject could detect obstacles as far away as $2-3 \mathrm{~m}$, with horizontal or vertical movements of the head the blindfolded test subject could understand the basic shape of objects without touching them, and the basic nature of the obstacles.
\end{abstract}

Copy Right, IJAR, 2016,. All rights reserved.

\section{Introduction:-}

The WHO projects that 285 million people are estimated to be visually impaired worldwide: 39 million are blind and 246 have low vision.About $90 \%$ of the world's visually impaired belong to the low income group. Scientists are trying to make eyes for the people who are blind. Some have even tried cathode ray implants inside the brain, but these are extremely expensive, provide very little vision and these procedures are invasive.

But what if we use another unconventional sense for sight? Bats can do it, dolphins can, why can't we? Echolocating animals emit calls out to the environment and listen to the echoes of those calls that return from various objects near them. They use these echoes to locate and identify the objects and obstacles.Since, about $90 \%$ of the world's visually impaired live in low-income settings, they can't afford anything but a walking stick that can't detect objects outside a 0.5 metre range or anything above waist height unless they don't collide with it.Basic spatial awareness is extremely important for any person, and therefore this device tries to solve the same problem. When the brain is deprived of input from one sensory organ, it can change in such a way that it augments other senses, a phenomenon called cross-modal neuroplasticity. 


\section{Methods and Improvements:-}

Approximately $90 \%$ of visually impaired people live in developing countries according to WHO projections. Since they the low cost is a very essential criterion, it is a must to make the device as economical as possible.

The initial research started off with analysing echolocation. Echolocation is the same as active sonar, using sounds made by the animal itself. Ranging is done by measuring the time delay between the animal's own sound emission and any echoes that return from the environment. The relative intensity of sound received at each ear as well as the time delay between arrival at the two ears provide information about the horizontal angle (azimuth) from which the reflected sound waves arrive. Echolocation had also been mastered by various humans too, who use clicks to find their way around, strengthening my hypothesis that soundscape based navigation was possible.

The selection of the correct sensor for the correct measurement of distance was extremely important because it needed to be cost effective, have a wide beam, and at the same time be able to detect versatile objects. In terms of accuracy, the infrared sensor was an obvious choice, but since it could only detect objects that weren't black, it wasn't used. I used an ultrasonic sensor (HC SR-04), which has a wider beam and can be used on all rigid bodies. Various positions were tested for the mounting of the sensor-

1.) When mounted on the chest it could just detect objects in front

2.) When mounted on the palm of the hand the direction the palm was pointing was proving too difficult to judge for the blindfolded test subject

3.) when mounted on the head, it gives the most swivel angle, hence was used.

The original idea was to have two units mounted on the other sides of the head to test the curvature of any object in front, however when conducting experiments, the pulses from both the sensors were interfering causing bogus values to be returned by the sensors. Hence the idea of having two sensors working seamlessly was abandoned.

\section{Results:-}

1.) Swivel angle covered by the system- $195^{\circ}$

2.) Values returned by the sensor- When graphed.

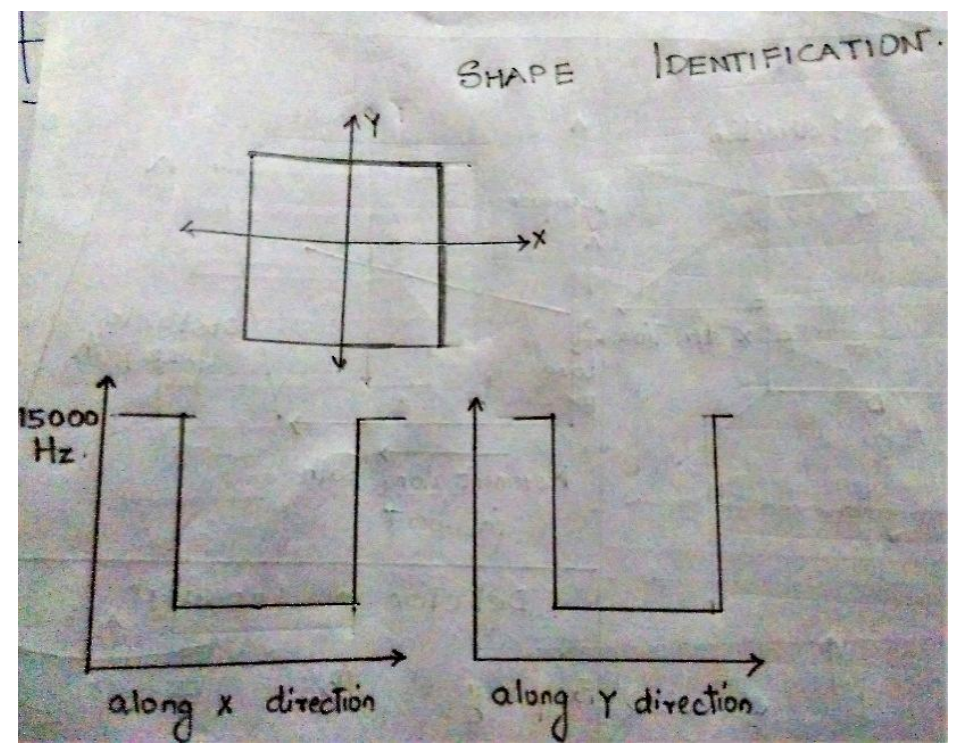

Figure 1. Test results for basic shape identification- SquareThe test was performed by holding a big square cardboard. 


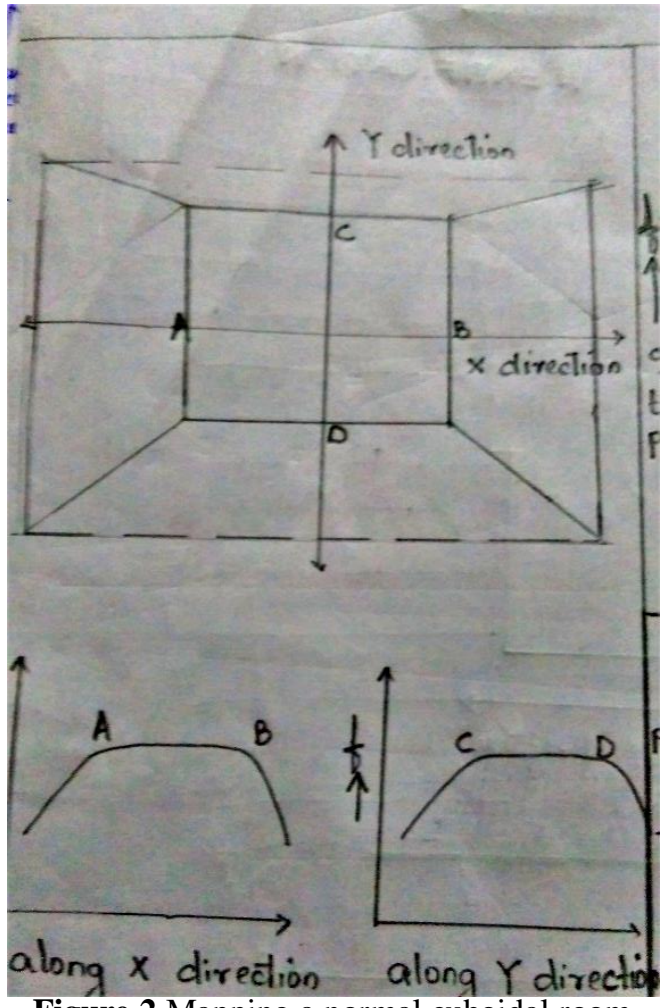

Figure 2.Mapping a normal cuboidal room

This test was carried out inside an empty cuboidal room to minimize interference from other objects. When the frequency of the tones produced are graphed with respect to head movement along a particular direction figure 2 is obtained.

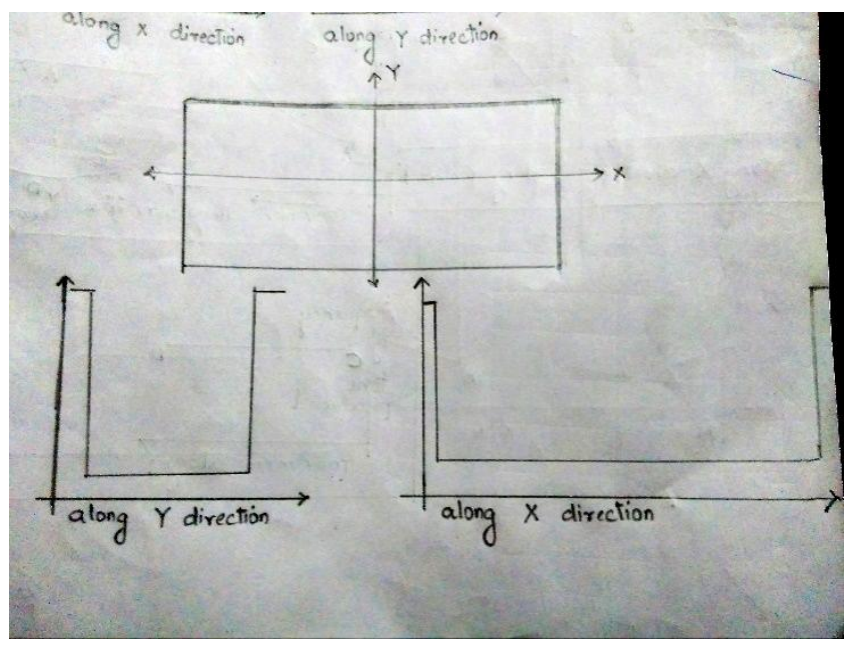

Figure 3:- Basic shape identification (rectangle) Test was performed by holding a big rectangular cardboard. 


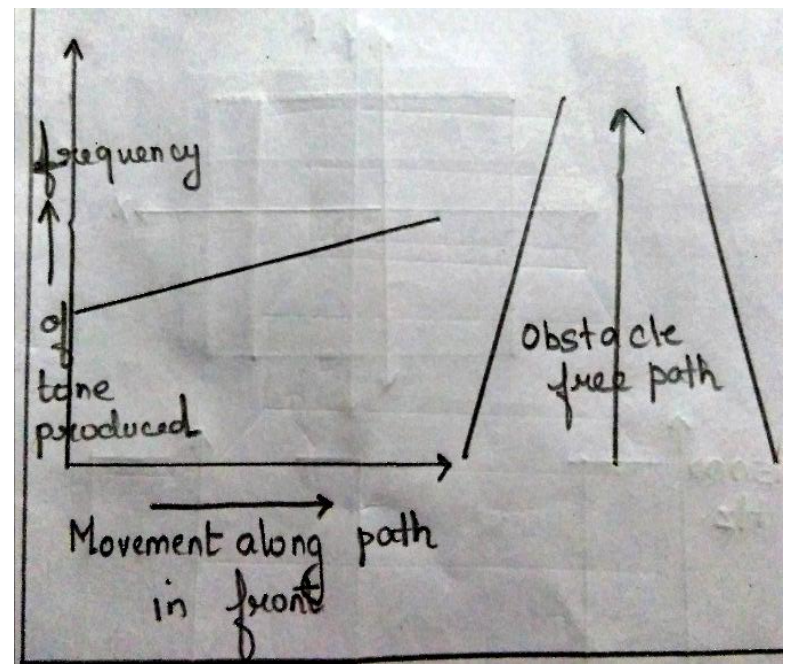

Figure 4:- Soundscape produced for an obstacle free path in front of the person, when the device is moving in forward direction (or the direction in which the person wants to move).

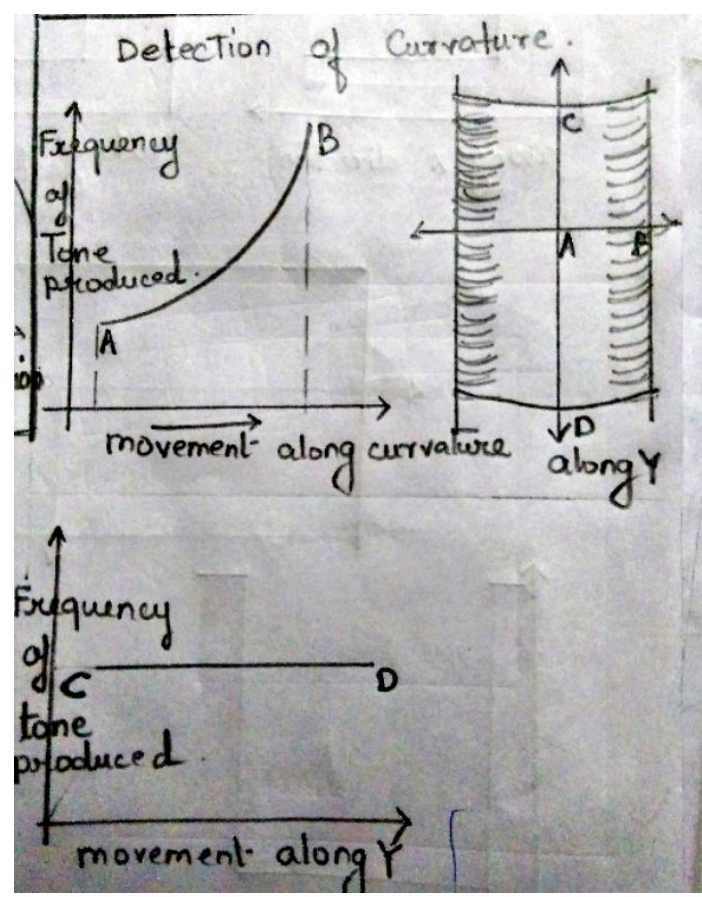

Figure 5:- Soundscape produced when a pillar is mapped-(cylindrical) -When the frequency of the tones produced are graphed with respect to head movement along $\mathrm{X}$ or $\mathrm{Y}$ axes. This test was used to detect the system's ability to detect curvature.

Additional tests with obstacles- The blindfolded test subject was introduced to an environment with obstacles, a normal car parking area. He was able to detect obstacles as far as 3 metres (In an unknown environment). Initial disorientation was observed. Experimental testing exposed flaws with the system such as few inaccurate values returned by the sensor and problems with detecting soft objects and sometimes amounts of non- comprehendible noise. This was primarily produced by pointing the sensor towards objects that are rapidly shifting in position, or many objects kept at a farawaydistance (caused by the beam angle). It also produced some unexpected results like being able to detect guide rods. 


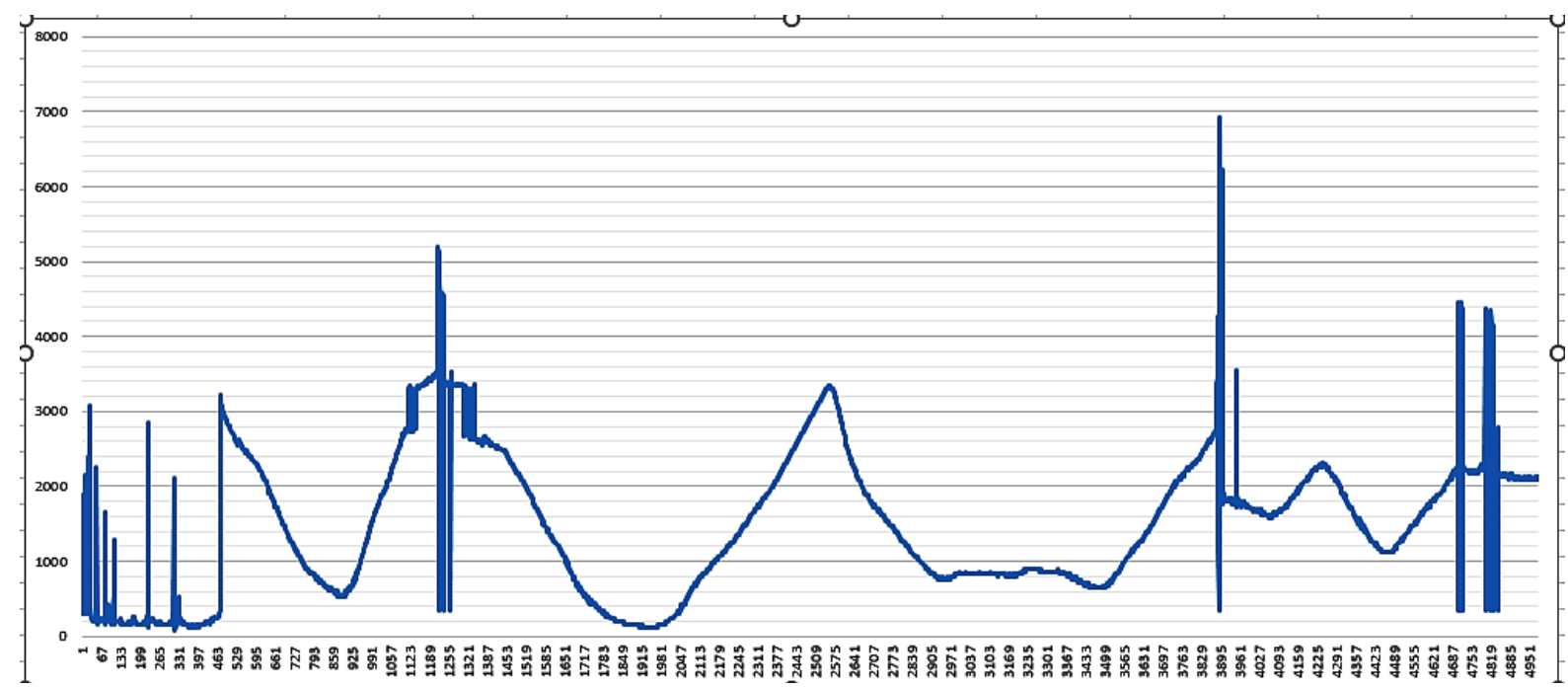

Figure 6. $\mathrm{X}$ axis- Movement of the module in whatever direction with time.Y axis - Frequency of the tone produced in Hertz

The above graph is an example of data feedback fromthe Bat-eye module - When Frequency is plotted against movement along various directions. This graph is from the values of frequency of tone sent from the Arduino board. *****Due to the huge amount of data feedback, segregation of data into parts that describe each shape couldn't be done, therefore the first few graphs were plotted by hand. ******

\section{Discussion And Conclusion:-}

The system could identify basic shapes, as well as give a person basic spatial awareness for navigation and obstacle avoidance at a low cost, as aimed, however, training for the blind people who are going to use the system (under controlled conditions) is necessary to understand what the tone feedback actually means to be able to use the device safely or to its fullest capabilities. Right now, It's imperative that further tests are carried out and extensive experimentation is done before the system is actually implemented as a substitute to the walking stick. It can also be used as a supplement to the standard walking stick.

\footnotetext{
Algorithm:-

\{Repeat these steps every 5 milliseconds\}

Step 1- Send pulse to Ultrasonic sensor

Step 2- Measure the time required for the sound wave to return sound

Step 4- Print the distance to the serial monitor

Step 5- Generate a frequency to correspond to the distance from the obstacle

Step 6- Play a tone on the speaker
}

Step 3-Calculate the distance to the obstacle based on the time required for the wave to return, and the speed of 


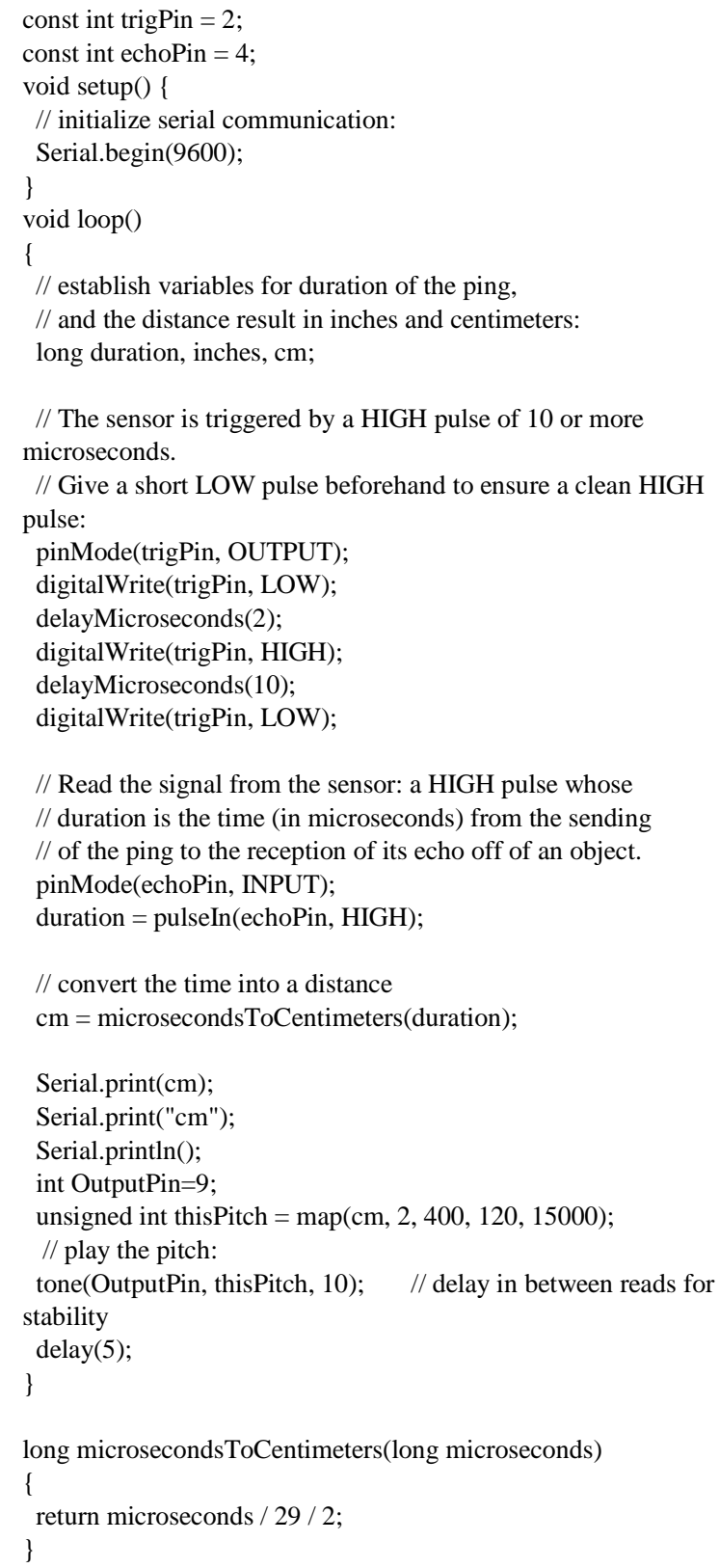

Acknowledgements:-

I would also like to thank my research mentor - DrKrishnendu Chatterjee for his constant help throughout this research.

\section{References:-}

Simmons, James A., M. Brock Fenton, and Michael J. O'Farrell. "Echolocation and pursuit of prey by bats." Science 203.4375 (1979): 16-21.

Kolb, Bryan, and Ian Q. Whishaw. "Brain plasticity and behavior." Annual review of psychology 49.1 (1998): 43-64.

Cohen, Leonardo G., et al. "Functional relevance of cross-modal plasticity in blind humans." Nature 389.6647 (1997): 180-183. 